\title{
Wireless Data Acquisition for Photovoltaic Power System
}

\author{
Makbul Anwari ${ }^{1}$, Arief Hidayat ${ }^{1}$, M. Imran Hamid ${ }^{1}$, and Taufik ${ }^{2}$ \\ ${ }^{1}$ Faculty of Electrical Engineering, Universiti Teknologi Malaysia, 81310 UTM Skudai, Johor, Malaysia \\ Electrical Engineering Department, Cal Poly State University, San Luis Obispo, USA \\ E-mail: makbul@ieee.org
}

\begin{abstract}
This paper presents a wireless system for monitoring the input and output of the array in a photovoltaic generation plant. The system comprises of sensors, data acquisition system, wireless access point and user computer that enable the users to access the array parameter wirelessly. Description and function of set up equipment are presented as well as the application program that supports the system.
\end{abstract}

\section{INTRODUCTION}

Recently, the number of energy in the world is reaching to concern state. This is caused by the need of energy is growing very fast. Due to concerns regarding global warming and air pollution, there has been an international movement in the promotion of renewable energy technologies for electricity generation, green energy is one among proposed solutions for these issues [1].

Solar energy is converted to electricity in a photovoltaic generation plant that contains photovoltaic array as solarelectricity conversion equipment, electrical power converter, power storage and other supporting equipment. According to operation mode, photovoltaic generation plants are met in isolation mode, grid interconnected mode and plant that can operate in both modes. For all of these modes, there are needs to acquire the input and output parameter of the photovoltaic array as the generation equipment. The acquired parameters are used for control action information, generation planning, energy forecasting, and performance observation or documentation need. Some of the parameters are irradiance, temperature and array's electrical output. A satisfied data acquisition system is required for this need.

Related to acquisition system for photovoltaic performance, Benghanem et al. have accomplished a research in which, several instruments are used to detect, integrate, and record solar energy measurement using both conventional electronics as well as microprocessor data acquisition system [2]. Further, Machacek et al., developed a system for measuring, collecting, analyzing, and displaying data for $100 \mathrm{~W}$ solar energy converter, data acquisition is formed by NI-6023E plug-in card and feed the rough data to the control program built in a MATLAB script [3].

Data from acquisition system module are needed to produce useful information. The speed of the process is the important parameter. To accommodate this requirement, during the past decade, digital control has been widely used. Digital control, which is determined by application of microprocessors, makes the sampling and computing process are faster than before. Implementation of such a system has been done on a dc voltage monitoring and control system for a wind turbine inverter [4].

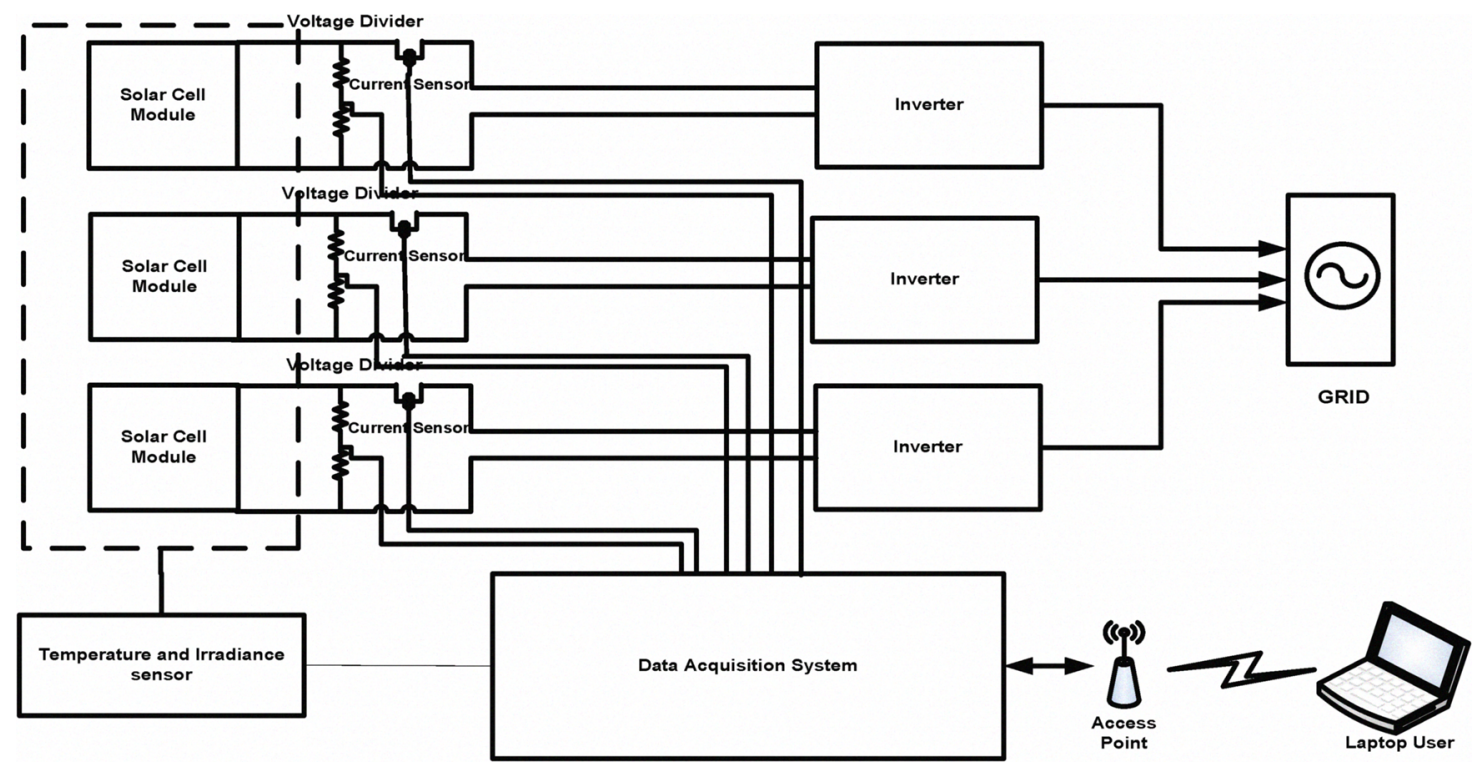

Fig 1. Simplified diagram of the wireless data acquisition for photovoltaic system. 
Regarding with data transmission, Chen,et.al, have studied carrying the acquired signal from data acquisition module using internet [1]. Java language is used for designing a dynamic webpage to graphically display various real time waveforms of the controlled system for multi-user at the same time. The system is implemented on a small scale wind power generation system equipped with an EZDSP 2812 controller. An FPGA ECIO is implemented as a bidirectional communication interface for coordinating the asynchronous data transmission modes.

In this paper, we present a photovoltaic generation monitoring system for a $5 \mathrm{kWp}$ laboratory scale photovoltaic generation. Temperature, irradiance, voltage, and current of the array are acquired, processed and then transmitted such that can be used for reviewing the performance of the generation plant. Acquired data is transmitted by wireless method using Wi-Fi signal (IEEE 802.11 standard), while microcontroller of PIC 16F877A is used to control the acquisition system process and a Delphi application program is built to graphically display the acquired data. Figure 1 show the simplified diagram of such system.

\section{PV GENERATION CHARACTERISTIC FOR MONITORING SYSTEM}

The acquisition system is aimed to detect and collect the parameters that indicate the electrical characteristic of the array and the factors influencing them.
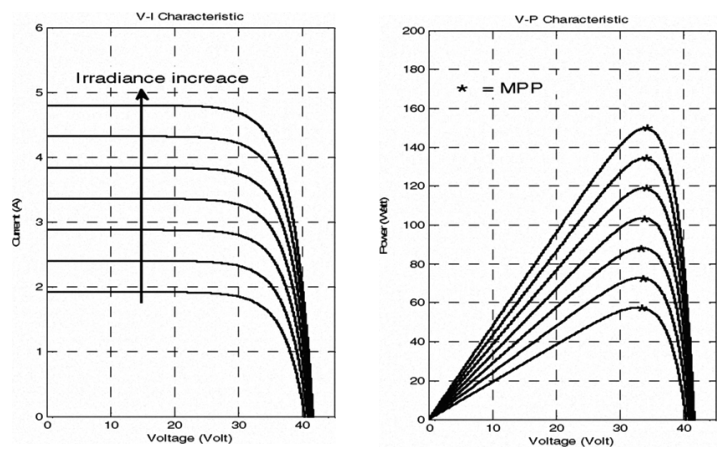

(a)
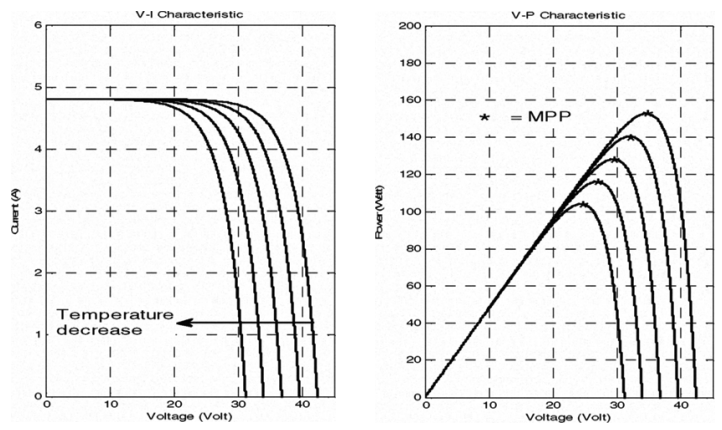

(b)

Fig. 2. V-I characteristic of PV module, (a) characteristics on various irradiance at a constant temperature, (b) Characteristic on various temperature at a constant irradiance
As shown in Figure 2, photovoltaic array characteristic ( $V-I$ curve) shows the dependency of cell current and voltage to irradiance and temperature. Irradiance contributes to the cell current, the higher irradiance the higher current draw by array photovoltaic, while the temperature effects to the cell voltage, the higher temperature the lower voltage appears on the cell terminal. In Figure 2a, it is shown a set of photovoltaic cell $I-V$ curve under varying irradiance at a constant temperature, meanwhile figure 2(b) shows the one at the same irradiance values, but under varying temperature. Both figures are also show the point where the multiplication of PV array voltage and current reaches the maximum value: maximum power point (MPP), at which condition that the array operates with maximum efficiency and produces maximum output power.

Variation of irradiance and temperature in photovoltaic module is characterized as short time fluctuation; follows the behavior of atmospheric condition around plant during time. The effect of this variation is the unpredictable variation of power output, current and voltage of the plant. An acquisition system for this condition should consider the phenomenon.

\section{PV GENERATION MONITORING SYSTEM}

The PV generation and monitoring system shown in Figure 1 , is a diagram of a laboratory scale system that contains three units photovoltaic array produces three dc voltages of $0-150$ range. These dc voltages is then fed to three single phase PV inverter respectively to be inverted to ac power before sending to the utility. Maximum current for each array are 10A dc. Array power input in form of temperature and irradiance and the dc output of array are picked up as data acquired for the monitoring system.

Three ACS754 current sensors with maximum current 50A and sensitivity of $37.8 \mathrm{mV} / \mathrm{A}$ are connected to dc output of each solar array, while irradiance and temperature sensor placed around the array. For voltage acquisition, $1 \mathrm{k} \Omega-1 \mathrm{M} \Omega$ voltage divider was used, which means $10 \mathrm{mV}$ for every one volt dc solar panel output. LM35 temperature sensor which sensitivity of $10 \mathrm{mV} /{ }^{\circ} \mathrm{C}$ is used for measure the ambient temperature of solar panel. The irradiance is sensed using LDR.

Data acquisition diagram is shown in Figure 3. In order to determine the analog signal from the sensor to be passed to $\mathrm{ADC}$, which contains eight analog signal from sensors ( 3 for dc current, 3 for dc voltage, one for irradiance and the remaining for temperature) dual eight-channels analog multiplexer DG407B are used. The analog multiplexer was controlled using microcontroller and passing the multiplexing signal with their own voltage references.

In the ADC block, each analog signal from multiplexer is digitalized to eight bits digital signal. Eight-bit signal is used caused by the condition that communication between microcontroller and serial to Ethernet module uses eight bit data. Thus, voltage reference $V_{r e f}$ for ADC is $V_{\text {ref }}=$ resolution $\times 2^{8}$, where the resolution is equal to sensitivity of each sensor. ADC operation is also controlled by the microcontroller.

In this system, the microcontroller is employed to run the 
following function: controlling the multiplexer for determining the analog signal from the sensor to be passed; controlling the operation of $\mathrm{ADC}$, and as communication protocol between Ethernet and the acquisition system. To accommodate these functions, the low-power consumption PIC 16F877A microcontroller is used. This unit is built within eight channels 10 bit ADC and an UART connection for serial communication.

Microcontroller serial connection is connected to Wiznet EGSR7150 in order to convert format data from serial to Ethernet data, conversion process is reversal. Further, the acquainted data is sent to the access point to be sent in form of Wi-Fi signal to connected user computer.

To accommodate communication between computer and acquisition system and to display the result, a computer application program is required.

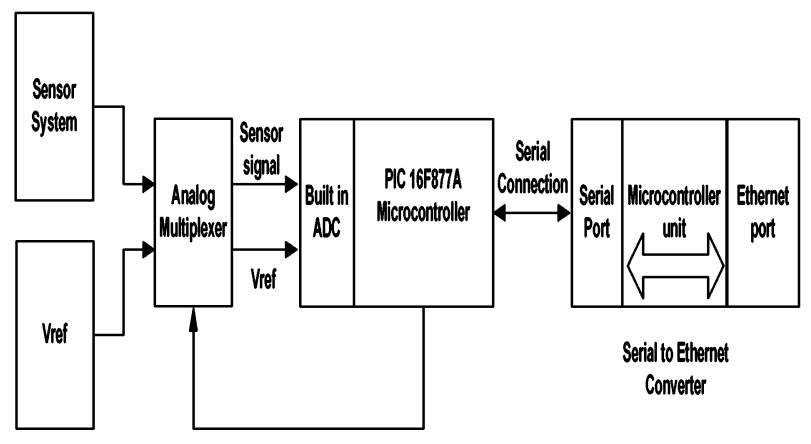

Fig. 3. Data acquisition system diagram

For this need, an application program, written in Delphi language is developed. This program is built to allow the user can interact and control the process steps in these two subsystems (microcontroller in acquisition system and displaying process in computer). Communication between computer and acquisition system is done wirelessly.

Communication process involves two application programs, one is in the computer side and the other is in microcontroller side. Flowchart of communication between these two application programs is shown in Figure 4, which shows two main blocks, indicates the process flow in each side.

First of all, the programs will self-initialize when they are activated. User can decide whether to acquire data or not. If acquisition data will be done, program will send a query command to the acquisition program on microcontroller contains which data to be acquired. Microcontroller in acquisition system -based on the command query- orders the multiplexer to by-pass the intended analog signal from the sensors and digitalized them.

Analog signal from the sensors contains high noise, which can degrade the measurement accuracy. To avoid this, analog signal is picked up and converted three times; their average is computed and become data to be sent back as acquisition data to user computer. In user computer, acquisition data is received by Ethernet port. Application program then processes the data to be displayed and to be saved in the virtual memory.

The displaying application program is shown in Figure 5.
There are 5 charts that show the measured and calculated parameters. Voltage, current, and calculated PV array power of whole channel is displayed in one chart respectively. Each PV array power output is obtained by multiplying the voltage and current of respective array. The array temperature and irradiance are displayed separately in others charts. Nominal values of these parameters are also displayed. To start data acquisition, user must connect the application to acquisition module by pressing the "Connect to network" button. Timer setting button is provided to determine measurement interval of the acquisition data.

Prototype of the photovoltaic acquisition system is shown in Figure 6. The left side picture shows layer for power supply, voltage divider and current sensor, this layer is placed in the bottom of acquisition compartment. The right side pictures shows layer for the microcontroller, multiplexer, $V_{\text {ref }}$ board and data converter.

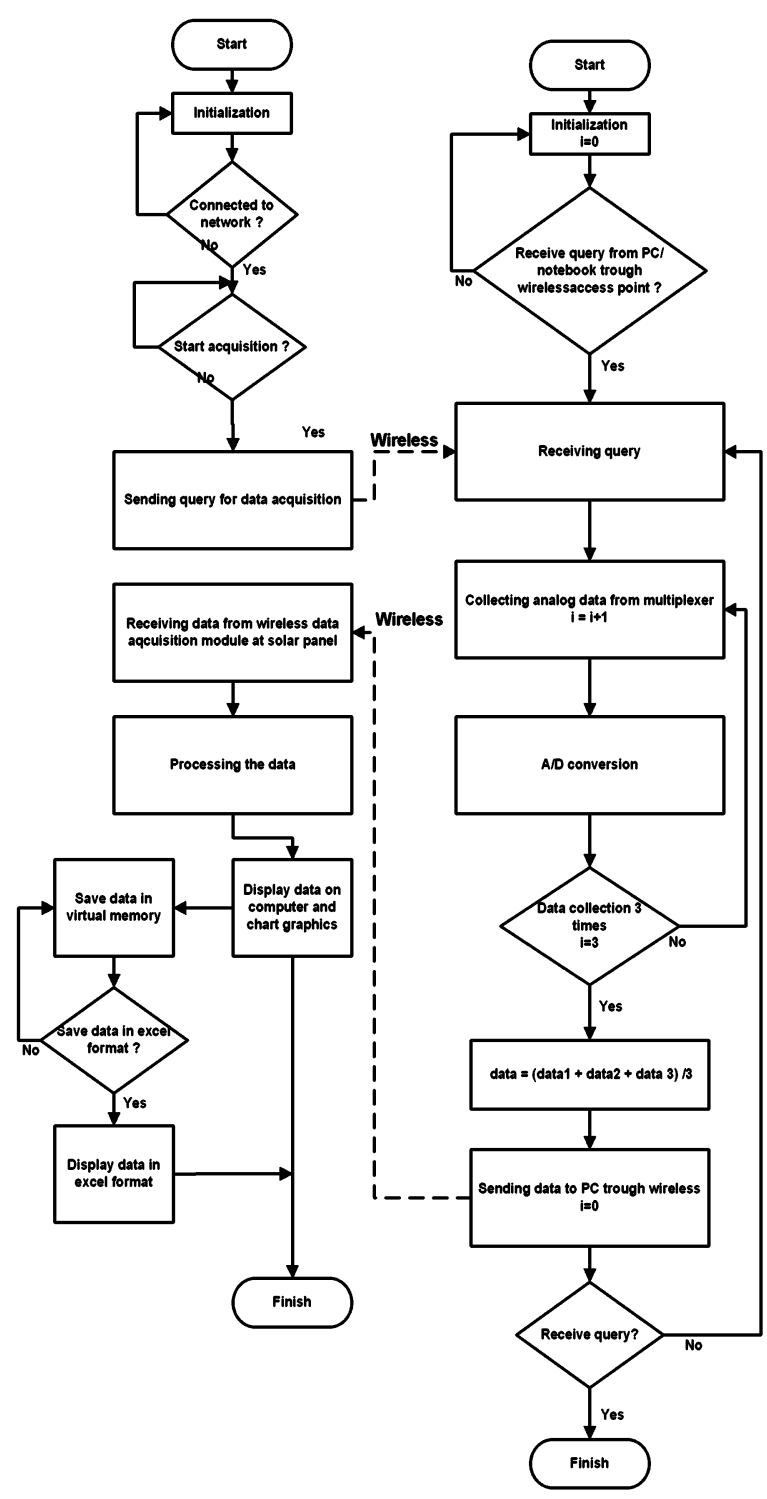

Fig. 4. PC and microcontroller software flowchart 


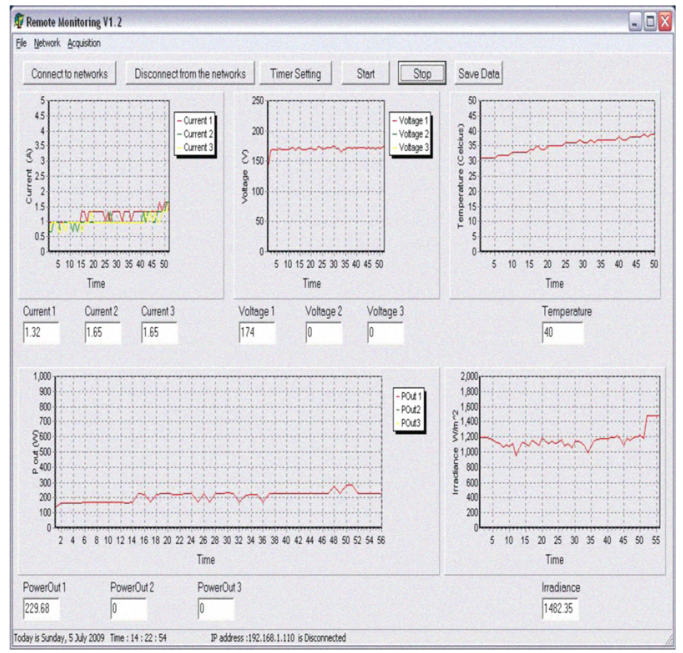

Fig.5. Application program appearance

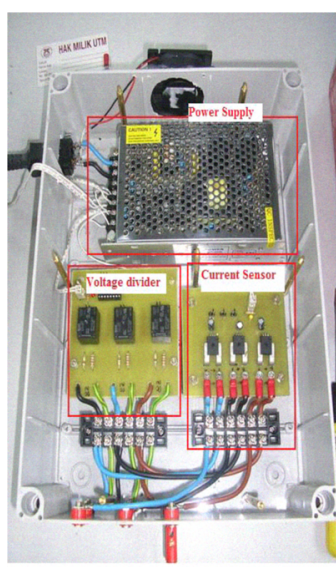

(a)

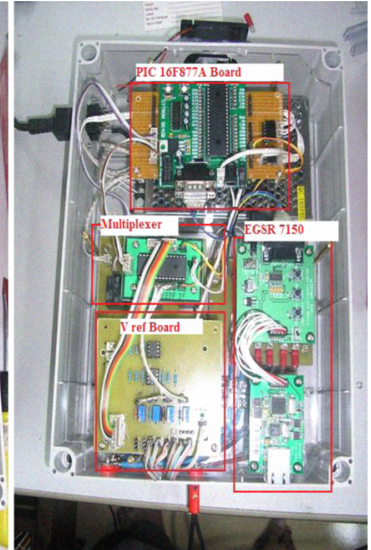

(b)
Fig. 6. Prototype of the photovoltaic acquisition system, (a) upper layer, (b) lower layer

\section{CONCLUSION}

A wireless data acquisition system for photovoltaic generation system that uses PIC16F877A microcontroller as the main control has been presented. Implementation of the EGSR7150 Ethernet to serial module and the access point as Wi-Fi communication tool between acquisition equipments and user computer for graphically displaying the acquired parameter has work properly as intended. Implementation of such a system on a laboratory scale photovoltaic generation shows the practical simplicity, efficient and low cost.

\section{V.ACKNOWLEDGMENT}

The authors thank the Malaysian Government, Ministry of Science, Technology and Innovation (MOSTI) for the Science Fund Grant, Project No. 01-01-06-SF0205.

\section{REFERENCES}

[1] Po-Yen Chen; Se-Kang Ho; Wei-Jen Lee; Chia-Chi Chu; Ching-Tsa Pan, "An Internet Based Embedded Network Monitoring System for Renewable Energy Systems", Proc. The 7th International Conference on Power Electronics, pp.225-228, October 22-26, 2007.

[2] M.Benghanem; A. Maafi, "Data Acquisition System for Photovoltaic Systems Performance Monitoring", IEEE Trans. on Instrumentation and Measurement, Vol.47, No.1, pp.30-33, February 2008.

[3] J. Machacek; z. Prochazka; J. Drapela, "System for Measuring and Collecting Data from Solar-cell Systems", Proc.9th International Conference Electrical Power Quality and Utilization, Barcelona, 9-11 October 2007.

[4] Z. Wang; L. Chang, "A DC voltage Monitoring and Control Method for Three Phase Grid-Connected Wind Turbine Inverters", IEEE Trans. On Power Electronics, Vol. 23, No.3, pp. 1118-1125, May 2008.

[5] T. Yuki; H. Yoshikiro; K. Kosuke, "Temperature and Irradiance Dependence of the I-V Curves of Various Kinds of Solar Cells" International Photovoltaic Science \& Engineering Conference, Shanghai, China 2005

[6] A. Moein, M. Pouladian, "WIH-Based IEEE 802.11 ECG Monitoring Implementation", Proceedings of the 29th Annual International Conference of the IEEE EMBS Cité Internationale, Lyon, France August 23-26, 2007.

[7] H. Zhao, X. Chen, K.H. Chon, "A Portable, Low-cost, Battery-powered Wireless Monitoring System for Obtaining Varying Physiologic Parameters from Multiple Subjects", Proceedings of the 28th IEEE EMBS Annual International Conference, New York City, USA, Aug 30Sept 3, 2006

[8] A. Perujo; R. Kaiser; D.U Sauer; H. Wenzl; I. Baring-Gould; N.Wilmot; F. Mattera; S. Tselepis; F

[9] H. Zhiqiang; Z. Wenxian; L. Jianke, "Research on High-Speed Data Acquisition and Processing Technique", The Eighth International Conference on Electronic Measurement and Instruments, ICEMI'2007 